\title{
Polycrystalline ZnO nanorods for lasing applications
}

Cite as: J. Appl. Phys. 125, 123103 (2019); https://doi.org/10.1063/1.5083965

Submitted: 02 December 2018 . Accepted: 10 March 2019. Published Online: 28 March 2019

N. I. Maad Tazri, O. L. Muskens (D), M. K. Shakfa (D), and W. Maryam (D)
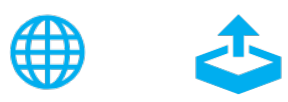

Export Citation

\section{ARTICLES YOU MAY BE INTERESTED IN}

A perspective on topological nanophotonics: Current status and future challenges

Journal of Applied Physics 125, 120901 (2019); https://doi.org/10.1063/1.5086433

\section{Bragg coherent diffractive imaging of strain at the nanoscale}

Journal of Applied Physics 125, 121101 (2019); https://doi.org/10.1063/1.5054294

Temporal evolution of photoinduced optical chirality in nanostructured light-sensitive waveguide thin films: Simultaneous excitation of $\mathrm{TE}_{\mathrm{O}}$ and $\mathrm{TE}_{1}$ modes

Journal of Applied Physics 125, 123101 (2019); https://doi.org/10.1063/1.5079506

\section{Applied Physics Reviews} Now accepting original research

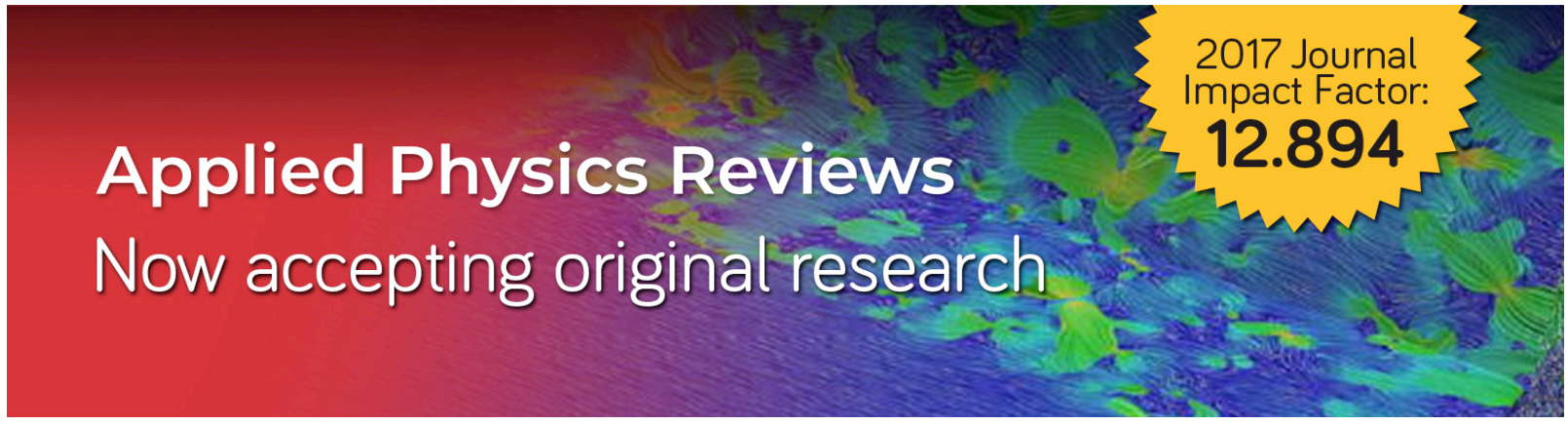




\title{
Polycrystalline ZnO nanorods for lasing applications
}

\author{
Cite as: J. Appl. Phys. 125, 123103 (2019); doi: 10.1063/1.5083965 \\ Submitted: 2 December 2018 . Accepted: 10 March 2019. \\ Published Online: 28 March 2019
}

N. I. Maad Tazri, ${ }^{7}$ O. L. Muskens, ${ }^{2}$ (D) M. K. Shakfa, ${ }^{3, a)}$ (iD and W. Maryam ${ }^{1, a) ~(D) ~}$

\author{
AFFILIATIONS \\ ${ }^{1}$ School of Physics, Universiti Sains Malaysia, 11800 Penang, Malaysia \\ ${ }^{2}$ Physics and Astronomy, Faculty of Physical Sciences and Engineering, University of Southampton, Southampton SO17 1BJ, \\ United Kingdom \\ ${ }^{3}$ King Abdullah University of Science and Technology (KAUST), Photonics Laboratory, Thuwal 23955-6900, Saudi Arabia
}

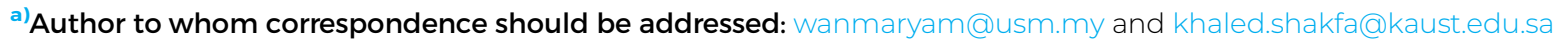

\begin{abstract}
Single and double mode random lasing were observed in a polycrystalline $\mathrm{ZnO}$ nanorod array. The double mode random lasing showed mode competition when the mode spacing was $2.3 \mathrm{~nm}$ or below. Structurally, X-ray diffraction measurements confirmed the formation of the polycrystalline phase, and photoluminescence measurements revealed a broad visible peak due to point defects, suggesting enhanced oxygen diffusion due to annealing. Our results suggest polycrystalline nanorods prepared by chemical bath deposition as a material system for obtaining random lasing for optoelectronic applications and devices.
\end{abstract}

Published under license by AIP Publishing. https://doi.org/10.1063/1.5083965

\section{INTRODUCTION}

The combination of gain and multiple scattering of light in a complex nanostructured media sets the scene for a range of phenomena including amplified stimulated emission and random lasing. ${ }^{1-3}$ In random lasers, the mirrors of a conventional laser are replaced by the multiple scattering material, which provides gain for a subset of modes in the complex medium. The balance between gain and scattering is subtle, and random lasing has been reported in both strongly and weakly scattering systems. ${ }^{4-9}$ In strongly scattering media, the mode competition generally results in multiple lasing peaks, which interact and compete for gain. ${ }^{10,11}$ However, to realize effective random-lasing-based optoelectronic applications like biosensors, integrated circuits, and diagnostics, it is inevitably important to control the random laser. There is no evidence of mode control, i.e., limiting the number of lasing modes as well as maintaining consistency in any of the aforementioned systems.

Several methods of controlling random lasing have been proposed, such as introducing point defects of polymer particles, ${ }^{12,13}$ controlling absorption, ${ }^{14}$ changing the Mie resonances, ${ }^{15}$ changing transport characteristics, ${ }^{16}$ waveguiding via electro-optic steering, ${ }^{17}$ and introducing some degree of disorder in $\mathrm{ZnO}$ nanorod arrays. ${ }^{18}$
The latter also showed that only annealed nanorod arrays can exhibit random lasing behavior. Random lasing in annealed $\mathrm{ZnO}$ microstructures in an argon atmosphere was reported, and annealing above $700{ }^{\circ} \mathrm{C}$ was required for lasing to occur. ${ }^{19}$ For the latter, however, all samples showed a crystalline structure for obtaining random lasing.

In this work, emission of single and double mode random lasing was obtained from polycrystalline $\mathrm{ZnO}$ annealed at a temperature of $500{ }^{\circ} \mathrm{C}$. Annealing above this value makes $\mathrm{ZnO}$ nanorods crystalline, and lasing characteristics from them are reported in Ref. 18. Random lasing in our current studies was achieved in a thin layer of strongly scattering polycrystalline $\mathrm{ZnO}$ nanowires. $\mathrm{X}$-ray diffraction measurements confirm that polycrystalline behavior and photoluminescence measurements revealed a broad visible peak possibly from oxygen interstitial due to annealing. Optical scattering properties of the layer are quantified using total transmission and reflection measurements, yielding information on the transport mean free path and showing that the nanorods mat is highly scattering. Single mode lasing emission showed consistency with very small lasing-emission wavelength changes across the sample. For double mode emission, there is a correlation between mode spacing and mode competition. 


\section{METHODS}

$\mathrm{ZnO}$ nanostructures were prepared on a pre-coated glass substrate by chemical bath deposition (CBD). All chemicals were used without further purification, and the aqueous solution was prepared using deionized water. The glass substrate was pre-coated with a $100 \mathrm{~nm}$ thick $\mathrm{ZnO}$ thin film using radio frequency (RF) magnetron sputtering. The substrate undergoes annealing treatment at $500{ }^{\circ} \mathrm{C}$ for $1 \mathrm{~h}$ in an oxygen environment with a flow rate of $3 \mathrm{sl} / \mathrm{m}$. The CBD solution was prepared with a mixture of $0.05 \mathrm{M}$ zinc nitrate $\left[\mathrm{Zn}\left(\mathrm{NO}_{3}\right)_{2} \cdot 6 \mathrm{H}_{2} \mathrm{O}\right]$ and $0.05 \mathrm{M}$ hexamethylenetetramine (HMT). The substrate was placed vertically inside the beaker containing the solution. The solution was then heated for $4 \mathrm{~h}$ at $96^{\circ} \mathrm{C}$. Finally, the sample undergoes repeated annealing with the same pre-growth annealing condition. Annealing at a temperature of $500^{\circ} \mathrm{C}$ results in a polycrystalline state of the nanorods' mats.

To investigate the structural properties, a field emission scanning electron microscope (FESEM) was used to view the morphology of the sample, and X-ray diffraction (XRD) was used to determine the crystal structure of the nanorods. Continuous-wave photoluminescence (cw-PL) measurements using an $\mathrm{HeCd}$ laser source operating at $325 \mathrm{~nm}$ were utilized to identify the optical properties of the sample. Raman measurements were also performed using an $\mathrm{Ar}^{+}$ion laser source to examine the structural properties. Transmittance and reflectance measurements were performed using a Fianium SC-400 supercontinuum light source, whereby total transmission and reflectance spectra are obtained utilizing an integrating sphere. Random lasing measurements were performed using a micro-PL system with an Nd:YAG pulsed laser source operating at $266 \mathrm{~nm}$ at $20 \mathrm{KHz}$ repetition rate and with a 550 ps pulse width. The beam spot size was $8 \mu \mathrm{m}$ in diameter with an incident peak power of $5 \mathrm{MW} \mathrm{cm}^{-2}$. The lasing emission was collected by a spectrometer with a resolution of $0.1 \mathrm{~nm}$. All measurements were performed at room temperature.

\section{MATERIAL CHARACTERISTICS}

Figure 1(a) shows an FESEM image of the sample with an inset showing the cross section. A good formation of nanorods with a hexagonal wurtzwite structure and a population that spreads equally on the surface are observed. The average diameter of the nanorods is $46 \pm 0.3 \mathrm{~nm}$, while the polydisperse distribution of nanorods has a standard deviation of around $10 \mathrm{~nm}$. The population density based on the FESEM image is roughly 255 nanorods $/ \mu \mathrm{m}^{2}$. Figure $1(\mathrm{~b})$ shows an XRD spectrum of the sample with the polycrystalline structure (JCPDS card no. 36-1451) of the hexagonal wurtzite structure. The peak at $2 \theta \sim 31^{\circ}, 34.4^{\circ}$, and $36^{\circ}$ corresponds to the (100), (002), and (101) planes, respectively. No peaks related to impurities are present. The inset of Fig. 1(b) shows the composition of the elements in the sample. Figure 1(c) shows a Raman spectrum obtained from
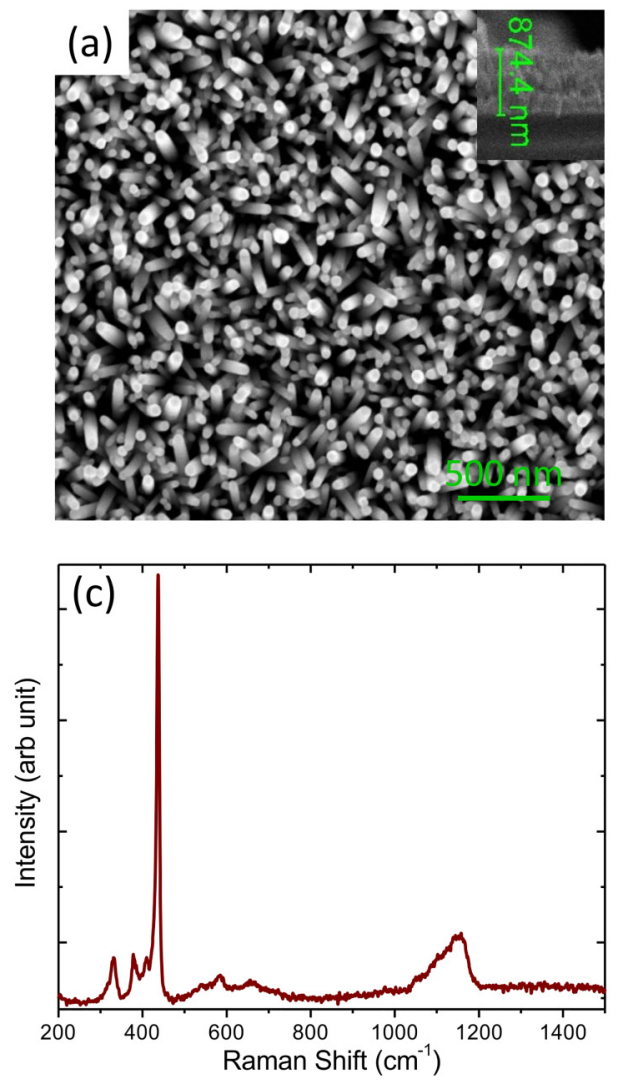
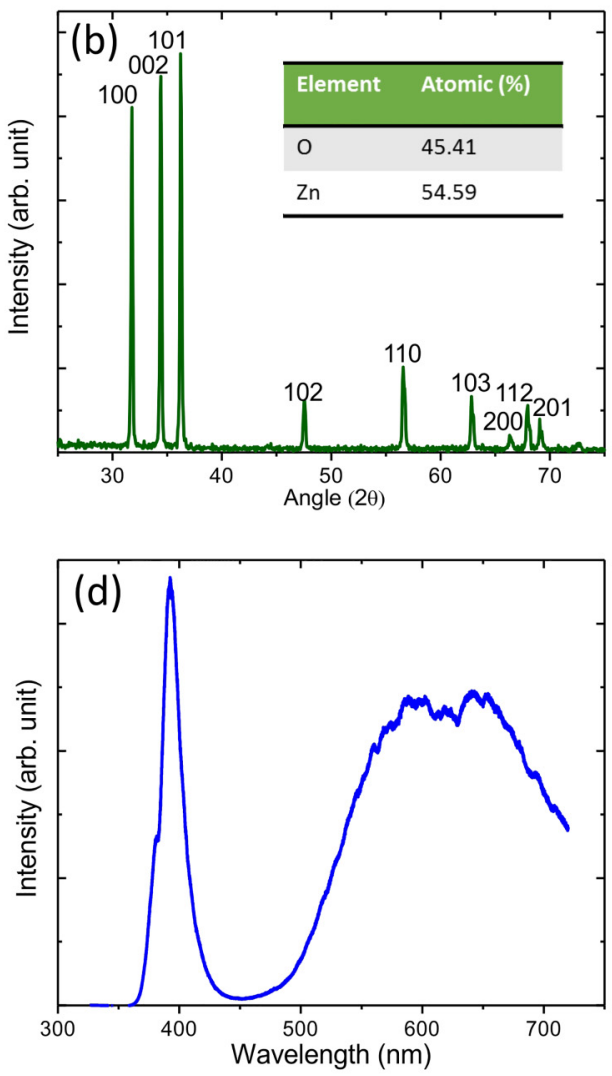

FIG. 1. (a) FESEM image of $\mathrm{ZnO}$ nanorods with inset showing the cross section. (b) XRD spectrum showing three dominant peaks for (100), (002), and (101) planes with inset showing the composition of the elements obtained from energy dispersive X-ray spectroscopy (EDX), (c) Raman spectrum with a peak at $435 \mathrm{~cm}^{-1}$ corresponding to $E_{2}(H)$, and (d) $C W-P L$ spectrum with near band edge emission centered at $394 \mathrm{~nm}$ and emission at $614 \mathrm{~nm}$ related to interstitial defects. 
the sample. The $\mathrm{E}_{2}(\mathrm{H})$ peak at $435 \mathrm{~cm}^{-1}$ is attributed to the hexagonal wurtzite structure and depends on the morphology of nanostructures. ${ }^{20}$ The $\mathrm{E}_{1}(\mathrm{~L})$ weak peak located at $587 \mathrm{~cm}^{-1}$ corresponds to the structural defect including oxygen vacancy. ${ }^{21}$ The peak at $332 \mathrm{~cm}^{-1}$ is attributed to $A_{1}(\mathrm{TO})$ due to anisotropy in the force constant, while the peak at $378 \mathrm{~cm}^{-1}$ is attributed to $\mathrm{E}_{2}(\mathrm{H})-\mathrm{E}_{2}(\mathrm{~L})$ due to the second order Raman spectrum from zoneboundary phonons. ${ }^{22}$ The relatively high intensity of the $\mathrm{E}_{2}(\mathrm{H})$ peak can be associated with bond breakage in $\mathrm{ZnO}$ due to defects. ${ }^{23}$ Figure 1(d) shows a PL spectrum of the sample; the emission at $394 \mathrm{~nm}$ is the near band edge (NBE) emission and the emission centered at $614 \mathrm{~nm}$ is typically attributed to O-vacancies and O-interstitial. ${ }^{24}$ The green emission is obviously broad due to interstitial and consequently affects the NBE emission. ${ }^{25}$

\section{TRANSPORT CHARACTERISTICS}

High density nanorods' mats made from high refractive index materials can exhibit strong optical scattering characteristics. ${ }^{26}$ Quantitative studies of the optical response of strongly scattering slabs can be used to extract their multiple scattering characteristics. ${ }^{27,28}$ Figure 2 shows total transmittance and reflectance spectra of the sample collected in an integrating sphere as well as coherent transmission that was obtained without the integrated sphere. Both total reflectance and transmittance spectra show a value of about $40 \%$ at $420 \mathrm{~nm}$ wavelength, indicating that at most $20 \%$ of light is absorbed by the sample in this range. This is an overestimate as some light is trapped by guided modes in the substrate and cannot be collected by the integrating sphere. The amount of light coherently transmitted is $6 \pm 1 \%$. This represents the amount of light not scattered within the structure and may be used to estimate the scattering mean free path using the Beer-Lambert law

$$
T_{\mathrm{COH}}=e^{-\frac{L}{l_{s}}}
$$

Here, $T_{\mathrm{COH}}$ is the coherent transmission, $L$ is the nanorod length, and $l_{s}$ is the scattering mean free path. From the coherent transmission, we estimate a value of $l_{s}=311 \pm 9 \mathrm{~nm}$. The transport mean free path can be obtained from the total transmission through the following relation: ${ }^{27}$

$$
T_{T O T}=\frac{1+\tau_{e}}{L / l_{t}+2 \tau_{e}},
$$

where $\tau_{e}$ is the extrapolation factor taking into account internal reflections at the boundaries of the layer, and $\tau_{e}=2 / 3$ corresponds to perfect index matching. ${ }^{29,30}$ The value of $\tau_{e}$ is of order unity in the case of a $\mathrm{ZnO}$ nanorods layer. From the experimental total transmission, we find a value of $l_{t}=292 \pm 13 \mathrm{~nm}$, which is of the same order as the scattering mean free path. This value is also of the same order for porous GaP networks, ${ }^{27}$ a material that was used in other random laser experiments showing spikes. ${ }^{11}$ Comparison of the transport mean free paths in other random lasers is provided in Ref. 31. The overall thickness of the nanorods layer in our sample is around 3 transport mean free paths.

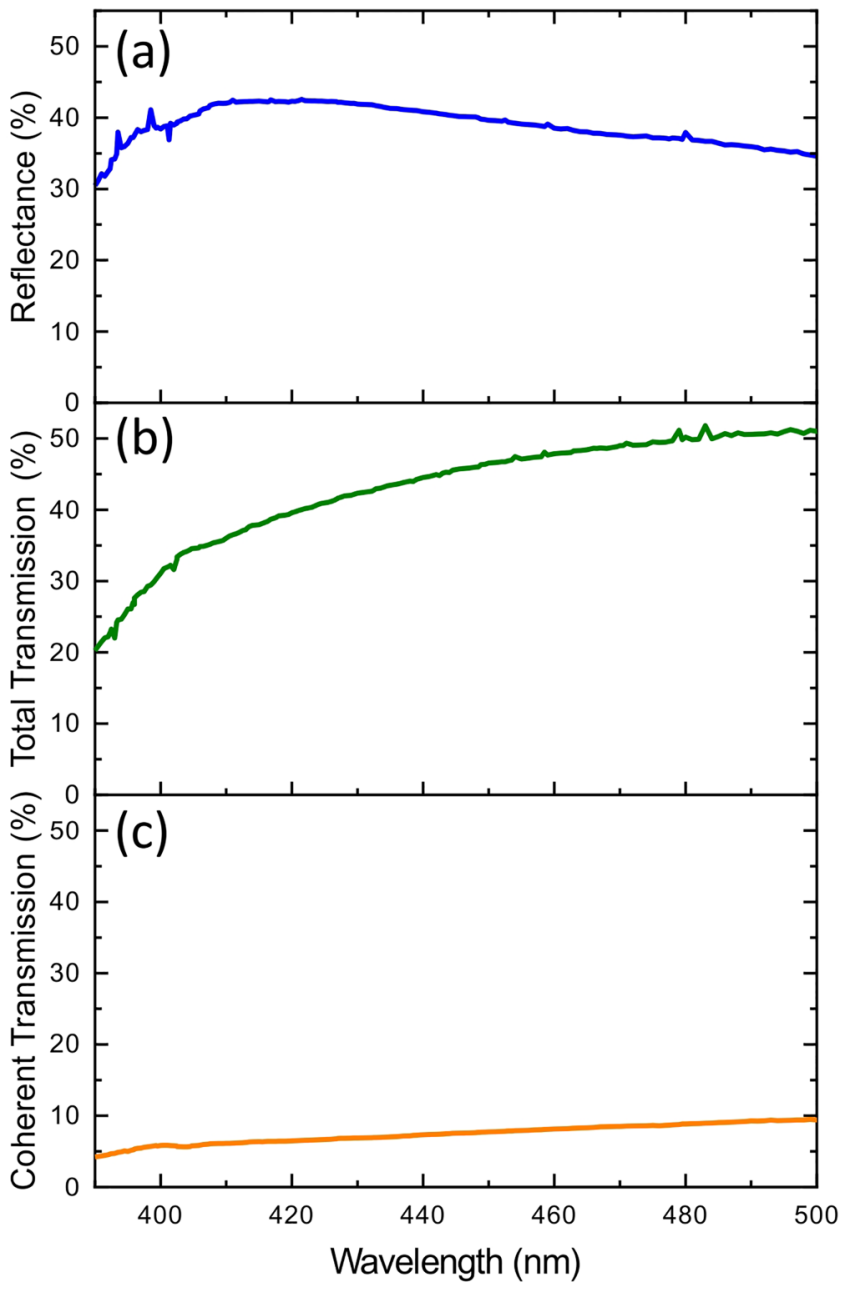

FIG. 2. Total reflectance, transmission, and coherent transmission (without integrating sphere) of the sample. About $40 \%$ of total reflectance and total transmission was obtained, and only about $6 \%$ of light was coherently transmitted.

\section{LASING CHARACTERISTICS}

Optical pumping measurements were performed to study random lasing emission from the sample with a pump beam spotsize of about $8 \mu \mathrm{m}$ in diameter and an incident peak power of $5 \mathrm{MW} \mathrm{cm}^{-2}$. From FESEM images in Fig. 1(a), about 3200 nanorods are involved in the optically pumped area. Figure 3(a) shows single mode random lasing from various positions of the sample. In some positions, double mode lasing is observed as shown in Fig. 3(b). Similar to the single modes, small shifts in wavelengths are observed. However, gain competition between the modes depended on their mode spacing. For example, a mode spacing of $6 \mathrm{~nm}$ showed a relative intensity of about 1 , indicating that the gain is shared almost equally between the two modes. When the modes are closer to each other, one mode becomes more dominant than the other. Also 

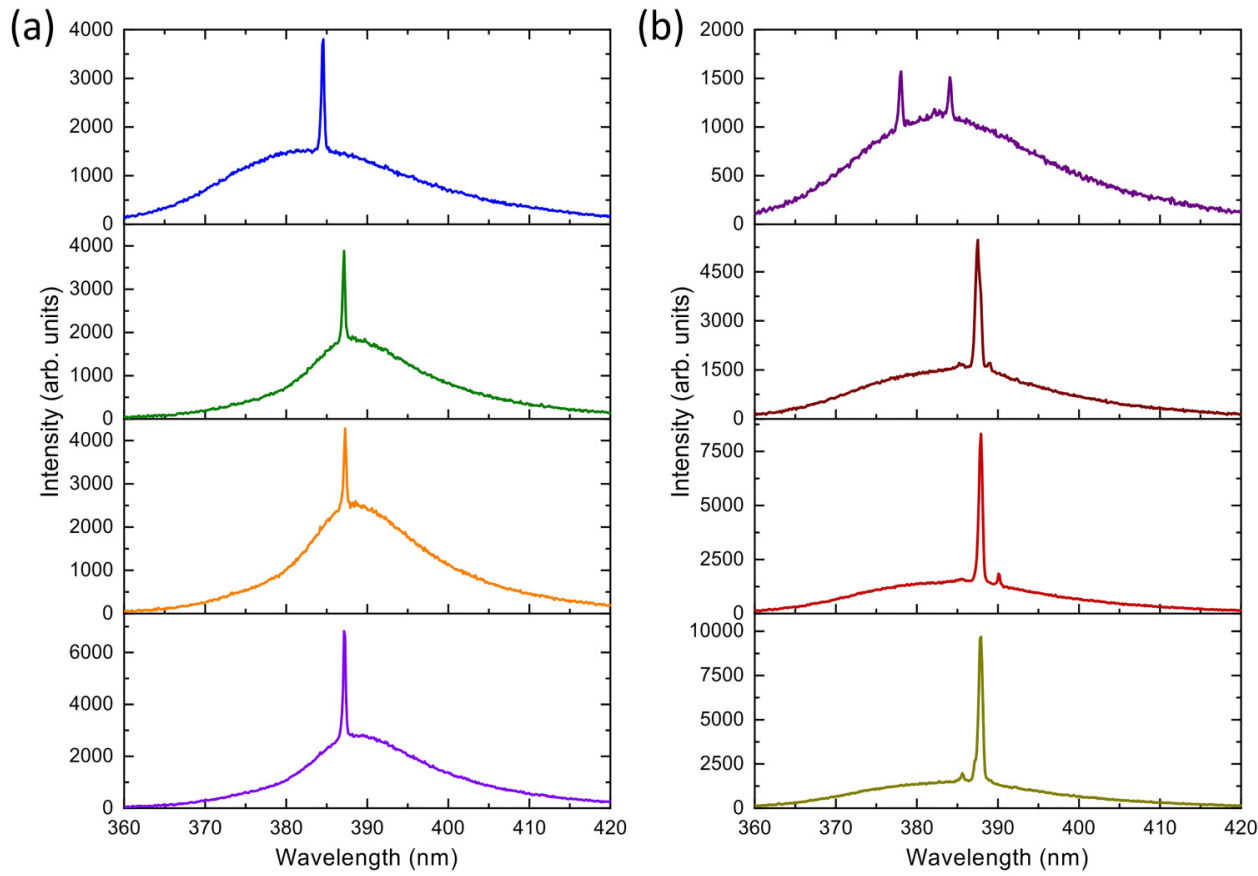

FIG. 3. (a) Single mode lasing at different sample's positions. Despite slight shifts in the lasing wavelength and varying lasing intensities, all modes maintained an FWHM of $0.35 \mathrm{~nm}$ at all positions. (b) Double mode random lasing at different positions. When mode spacing was $6 \mathrm{~nm}$ (top), an equal distribution of the gain between modes is observed, whereas in all other cases where mode spacing was $2.3 \mathrm{~nm}$ or below, gain competition observed with one mode is dominant over the other. observed is that the dominant modes have a larger FWHM, whereas in the case of equal emission the FWHM of the lasing mode is almost the same. It is possible that having an array of $\mathrm{ZnO}$ vertical nanowires for random lasing instead of $\mathrm{ZnO}$ spheres or microresonators, as most reports for random lasing, changes the scattering of the light and hence the gain profile. In this regime, lasing is either single mode or double mode depending on changes of the transport mean free path on the sample. There is also an indication that point defects play a significant role in observing lasing as undoped samples did not show any lasing emission. This may provide a possibility of limiting the number of modes in a random laser as well as for modelocking applications. A summary of the lasing characteristics of double mode emission is provided in Table I.

The short mean free path of light inside the nanorods mat rules out the formation of cavity modes by multiple interference in the slab. To further exclude possible effects from a Fabry-Perot

TABLE I. Characteristics of the double lasing modes at specific sample positions.

\begin{tabular}{lcccc}
\hline \hline Position & $\begin{array}{c}\text { Wavelength } \\
(\mathbf{n m})\end{array}$ & $\begin{array}{c}\text { FWHM } \\
(\mathbf{n m})\end{array}$ & $\begin{array}{c}\text { Mode spacing } \\
(\mathbf{n m})\end{array}$ & $\begin{array}{c}\text { Peak intensity } \\
\text { ratio }\end{array}$ \\
\hline 1 & 378.0 & 0.43 & 6.0 & 1.04 \\
& 384.0 & 0.47 & & \\
2 & 387.5 & 0.76 & 2.3 & 0.31 \\
3 & 385.2 & 0.21 & & \\
& 388.0 & 0.41 & 2.0 & 0.22 \\
4 & 390.0 & 0.29 & & \\
& 385.6 & 0.29 & 2.3 & 0.21 \\
\hline \hline
\end{tabular}

cavity formed by the nanorods mat, we estimated the expected behavior for such a cavity. From a simple estimation of the mode spacing in Fabry-Perot systems follows: ${ }^{32}$

$$
\Delta \lambda=\lambda^{2} /(2 n L)
$$

where $L$ is the cavity length, $n$ is the refractive index (2.45), and $\lambda$ is the resonator wavelength. The average height of the nanorod is 850 $\mathrm{nm}$, and correspondingly, the mode spacing should be $35.4 \mathrm{~nm}$. However, the mode spacing, in Table I, varies only between $2 \mathrm{~nm}$ and $6 \mathrm{~nm}$, indicating that the detected emission is not due to the Fabry-Perot effect. Another possible lasing mechanism is four wave mixing and whispering gallery modes. However, the diameter of our nanorods is too small (less than $200 \mathrm{~nm}$ ) for this to occur especially in its current resonator structure. . $^{3-35}$

The nature of random lasing in polycrystalline $\mathrm{ZnO}$ nanorods' mats could be further investigated in future studies, for example, by looking at the spatial extent of lasing modes ${ }^{10,36}$ and studying its dependence on sample parameters such as the thickness of the mat, the alignment of the nanorods, and the role of the polycrystalline structure. Our work is a first step toward implementation of chemical bath synthesis as a low-cost scalable technique for producing random laser materials, which could spark new applications such as optical sensors. ${ }^{37}$

\section{CONCLUSION}

Single and double mode random lasing within a strong scattering regime were observed in thin layers of polycrystalline $\mathrm{ZnO}$ nanorods. Double modes showed mode competition when the mode spacing was $2.3 \mathrm{~nm}$ and below. When the mode spacing was $6 \mathrm{~nm}$, the two emission peaks show almost equal intensity as well as the same 
FWHM. Our findings overall demonstrate the possibility of utilizing the scattering regime in $\mathrm{ZnO}$ as a control method for random lasers applications. We believe that our findings spark more experimental and theoretical works in looking at the effects of grain and structural boundaries on random lasing.

\section{ACKNOWLEDGMENTS}

The authors are grateful for partial funding from a USM shortterm grant (No. 304/PFIZIK/6312155) and from the Global Challenges Research Fund (GCRF)-EPSRC Institutional Sponsorship. W. Maryam also acknowledges Professor Azlan Abdul Aziz for in-kind contribution of the sputtering target. The authors would like to thank Professor Dr. Boon S. Ooi from King Abdullah University of Science and Technology (KAUST) for giving the opportunity to perform the random-lasing measurements in his laboratory (Photonics Laboratory).

\section{REFERENCES}

${ }^{1}$ H. Cao, J. Y. Xu, S.-H. Chang, and S. T. Ho, "Transition from amplified spontaneous emission to laser action in strongly scattering media," Phys. Rev. E 61(2), 1985-1989 (2000).

${ }^{2}$ C.-S. Wang, T.-Y. Chang, T.-Y. Lin, and Y.-F. Chen, "Biologically inspired flexible quasi-single-mode random laser: An integration of Pieris canidia butterfly wing and semiconductors," Sci. Rep. 4(1), 6736 (2015).

${ }^{3}$ H. Fujiwara, T. Suzuki, R. Niyuki, and K. Sasaki, "ZnO nanorod array random lasers fabricated by a laser-induced hydrothermal synthesis," New J. Phys. 18(10), 103046 (2016).

${ }^{4}$ W. Z. W. Ismail, D. Liu, S. Clement, D. W. Coutts, E. M. Goldys, and J. M. Dawes, "Spectral and coherence signatures of threshold in random lasers," J. Opt. 16(10), 105008 (2014)

${ }^{5}$ X. Meng, K. Fujita, S. Murai, and K. Tanaka, "Coherent random lasers in weakly scattering polymer films containing silver nanoparticles," Phys. Rev. A 79(5), 053817 (2009).

${ }^{6} \mathrm{X}$. Wu, et al., "Random lasing in weakly scattering systems," Phys. Rev. A 74(5), 053812 (2006).

${ }^{7}$ R. C. Polson and Z. V. Vardeny, "Organic random lasers in the weak-scattering regime," Phys. Rev. B 71(4), 045205 (2005).

${ }^{8}$ M. Sakai, et al., "Random laser action in GaN nanocolumns," Appl. Phys. Lett. 97(15), 151109 (2010).

${ }^{9}$ C. Tolentino Dominguez, M. d. A. Gomes, Z. S. Macedo, C. B. de Araújo, and A. S. L. Gomes, "Multi-photon excited coherent random laser emission in $\mathrm{ZnO}$ powders," Nanoscale 7(1), 317-323 (2015).

${ }^{10}$ K. L. van der Molen, R. W. Tjerkstra, A. P. Mosk, and A. Lagendijk, "Spatial extent of random laser modes," Phys. Rev. Lett. 98(14), 143901 (2007).

${ }^{1}$ M. Noginov, J. Novak, and S. Williams, "Modeling of photon density dynamics in random lasers," Phys. Rev. A 70(6), 063810 (2004).

${ }^{12}$ R. Niyuki, H. Fujiwara, Y. Ishikawa, N. Koshizaki, T. Tsuji, and K. Sasaki, "Toward single-mode random lasing within a submicrometre-sized spherical ZnO particle film," J. Opt. 18(3), 035202 (2016).

${ }^{13}$ H. Fujiwara, R. Niyuki, Y. Ishikawa, N. Koshizaki, T. Tsuji, and K. Sasaki, "Low-threshold and quasi-single-mode random laser within a submicrometersized ZnO spherical particle film," Appl. Phys. Lett. 102(6), 061110 (2013).

${ }^{14}$ R. G. S. El-Dardiry and A. Lagendijk, "Tuning random lasers by engineered absorption," Appl. Phys. Lett. 98(16), 161106 (2011).

${ }^{15} \mathrm{M}$. Gaio, M. Peruzzo, and R. Sapienza, "Tuning random lasing in photonic glasses," Opt. Lett. 40(7), 1611 (2015).
${ }^{16}$ N. Bachelard, P. Gaikwad, R. Backov, P. Sebbah, and R. A. L. Vallée, "Disorder as a playground for the coexistence of optical nonlinear effects: Competition between random lasing and stimulated Raman scattering in complex porous materials," ACS Photonics 1(11), 1206-1211 (2014).

${ }^{17}$ G. Assanto, S. Perumbilavil, A. Piccardi, and M. Kauranen, "Electro-optic steering of random laser emission in liquid crystals," Photonics Lett. Pol. 10(4), 103 (2018).

${ }^{18}$ W. Maryam, N. Fazrina, M. R. Hashim, H. C. Hsu, and M. M. Halim, "Optically driven random lasing in $\mathrm{ZnO}$ nanorods prepared by chemical bath deposition," Photonics Nanostruct. Fundam. Appl. 26, 52-55 (2017).

${ }^{19}$ T.-F. Dai, W.-C. Hsu, and H.-C. Hsu, "Improvement of photoluminescence and lasing properties in $\mathrm{ZnO}$ submicron spheres by elimination of surfacetrapped state," Opt. Express 22(22), 27169 (2014).

${ }^{20}$ E. Muchuweni, T. S. Sathiaraj, and H. Nyakotyo, "Synthesis and characterization of zinc oxide thin films for optoelectronic applications," Heliyon 3(4), e00285 (2017).

${ }^{\mathbf{2 1}} \mathrm{Y}$. Wang, et al., "Effect of different annealing atmospheres on the structure and optical properties of ZnO nanoparticles," J. Alloys Compd. 485(1-2), 743-746 (2009).

${ }^{\mathbf{2 2}}$ P. K. Samanta and A. K. Bandyopadhyay, "Chemical growth of hexagonal zinc oxide nanorods and their optical properties," Appl. Nanosci. 2(2), 111-117 (2012).

${ }^{23}$ S. A. Bidier, M. R. Hashim, and A. M. Aldiabat, "Effect of postannealing treatment on structural and optical properties of $\mathrm{ZnO}$ nanorods prepared using chemical bath deposition," J. Electron. Mater. 46(7), 4455-4462 (2017).

${ }^{\mathbf{2 4}} \mathrm{K}$. Vanheusden, W. L. Warren, C. H. Seager, D. R. Tallant, J. A. Voigt, and B. E. Gnade, "Mechanisms behind green photoluminescence in $\mathrm{ZnO}$ phosphor powders," J. Appl. Phys. 79(10), 7983-7990 (1996).

${ }^{25}$ L. L. Yang, Q. X. Zhao, M. Willander, J. H. Yang, and I. Ivanov, "Annealing effects on optical properties of low temperature grown ZnO nanorod arrays," J. Appl. Phys. 105(5), 053503 (2009).

${ }^{26}$ O. L. Muskens, S. L. Diedenhofen, B. C. Kaas, R. E. Algra, E. P. A. M. Bakkers, J. Gómez Rivas, and A. Lagendijk, "Large photonic strength of highly tunable resonant nanowire materials," Nano Lett. 9(3), 930-934 (2009).

${ }^{27}$ F. J. P. Schuurmans, "Strongly photonic macroporous gallium phosphide networks," Science 284(5411), 141-143 (1999).

${ }^{28}$ J. Gómez Rivas, R. Sprik, A. Lagendijk, L. D. Noordam, and C. W. Rella, "Static and dynamic transport of light close to the Anderson localization transition," Phys. Rev. E 63(4), 046613 (2001).

${ }^{29} \mathrm{D}$. J. Durian, "Influence of boundary reflection and refraction on diffusive photon transport," Phys. Rev. E 50(2), 857-866 (1994).

${ }^{30}$ J. X. Zhu, D. J. Pine, and D. A. Weitz, "Internal reflection of diffusive light in random media,” Phys. Rev. A 44(6), 3948-3959 (1991).

${ }^{31}$ K. L. van der Molen, A. P. Mosk, and A. Lagendijk, "Quantitative analysis of several random lasers," Opt. Commun. 278(1), 110-113 (2007).

${ }^{32}$ H.-C. Hsu, C.-Y. Wu, and W.-F. Hsieh, "Stimulated emission and lasing of random-growth oriented $\mathrm{ZnO}$ nanowires,” J. Appl. Phys. 97(6), 064315 (2005).

${ }^{33}$ G. Grinblat, Y. Li, M. P. Nielsen, R. F. Oulton, and S. A. Maier, "Degenerate four-wave mixing in a multiresonant germanium nanodisk," ACS Photonics 4(9), 2144-2149 (2017).

${ }^{34}$ C.-H. Lu, T.-Y. Chao, Y.-F. Chiu, S.-Y. Tseng, and H.-C. Hsu, "Enhanced optical confinement and lasing characteristics of individual urchin-like $\mathrm{ZnO}$ microstructures prepared by oxidation of metallic Zn," Nanoscale Res. Lett. 9(1), 178 (2014).

${ }^{35}$ O. Malik, K. G. Makris, and H. E. Türeci, "Spectral method for efficient computation of time-dependent phenomena in complex lasers," Phys. Rev. A 92(6), 063829 (2015).

${ }^{36} J$. Fallert, R. J. B. Dietz, J. Sartor, D. Schneider, C. Klingshirn, and H. Kalt, "Co-existence of strongly and weakly localized random laser modes," Nat. Photonics 3(5), 279-282 (2009).

${ }^{37}$ S. Caixeiro, M. Gaio, B. Marelli, F. G. Omenetto, and R. Sapienza, "Silk-based biocompatible random lasing,” Adv. Opt. Mater. 4(7), 998-1003 (2016). 